\title{
A novel disposable biosensor based on SiNWs/AuNPs modified-screen printed electrode for dengue virus DNA oligomer detection
}

\begin{abstract}
In this study, a disposable screen-printed gold electrode (SPGE) utilized of silicon nanowires (SiNWs) and gold nanoparticles as sensing material was fabricated for detection of DNA oligomers related to dengue virus. Firstly, SiNWs/AuNPs-SPGE was developed by the dispersion of SiNWs in 3-Aminopropyltriethoxysilane (APTES, $0.5 \%$ ) onto bare SPGE. Secondly, the AuNPs decoration on SiNWs-SPGE surface was functionalized using dithiopropionic acid (DTPA) through a self-assembly monolayer (SAM) technique. The electrochemical response of methylene blue (MB) as a redox indicator towards synthetic DNA oligomer after hybridization on SiNWs/AuNPs-SPGE was recorded by cyclic voltammetry (CV) and differential pulse voltammetry (DPV) techniques. The results demonstrated that the reduction peak current of MB was significantly decreased after DNA hybridization process. In addition, the developed biosensor showed a good storage stability and could achieve a linear range of $1.0 \times 10-11-1.0 \times 10-7 \mathrm{M}(\mathrm{R}=0.98)$ with the detection limit of $1.63 \times 10-12 \mathrm{M}$.
\end{abstract}

Keyword: Silicon nanowires; Gold nanoparticles; DNA hybridization; Dengue virus 